\title{
Characterisation of non-uniform functional surfaces: towards linking basic surface properties with electrocatalytic activity $\dagger$
}

\author{
Artjom Maljusch, ${ }^{a}$ John B. Henry, ${ }^{b}$ Jakub Tymoczko, ${ }^{\text {ab }}$ Aliaksandr S. Bandarenka ${ }^{* b}$ \\ and Wolfgang Schuhmann*ab
}

Functional materials, particularly heterogeneous catalysts, are often non-uniform at a microscopic level making their detailed characterisation extremely complex. This complexity inhibits the design and implementation of novel functional materials as such characterisation is a key to understanding interfaces for heterogeneous catalysis. We demonstrate that a combination of Scanning Kelvin Probe (SKP) and Scanning Electrochemical Microscopy (SECM) experiments made over the same sample surface using an integrated SKP-SECM system provides a powerful and robust tool to link basic surface properties with the observed electrocatalytic activity. As the SKP-response can be accurately assessed using modern quantum chemical approaches to benchmark analytical signals for different surface structures with varying compositions, application of an integrated SKP-SECM system can offer valuable insight into the origin of the observed electrocatalytic activity. As model objects, we used Pt(111)-like thin films modified with sub-monolayer and monolayer amounts of $\mathrm{Cu}$ atoms located at the electrode surface and in the sub-surface region. The exact position of the $\mathrm{Cu}$ atoms relative to the topmost $\mathrm{Pt}$ layer greatly affects basic surface properties and governs the electrocatalytic activity of the surface towards various reactions, i.e. the oxygen reduction reaction. SKP-SECM appeared to be a very sensitive tool to monitor those changes as a function of the spatial coordinates.

Received 15th October 2013

Accepted 6th November 2013

DOI: $10.1039 / c 3 r a 45845 h$

www.rsc.org/advances
The ideal combination of techniques should be both informative and affordable. Among those techniques the scanning Kelvin probe (SKP) is a relatively simple, but sensitive technique which can obtain valuable information about the surface status. ${ }^{16}$ For example, in the case of metals, SKP measures responses which are proportional to work function differences across the surface. These work function differences are a sensitive indicator of the surface condition, which can be affected by adsorbed layers, surface composition, structure, charging and the presence of contaminations. ${ }^{17}$ Importantly, work function differences can be calculated with quantum chemical approaches for a variety of surfaces with high accuracy. ${ }^{18,19}$ The relative ease in the ability to calculate and measure benchmark values which are proportional to SKP-values for various surfaces makes the scanning Kelvin probe a good candidate to complement electrochemical microscopy measurements.

In this work, we show how SKP-experiments, performed in the gas phase under close to ambient conditions, when combined with scanning electrochemical microscopy (SECM) measurements, performed over the same sample surface by using the same microelectrode as tip in both techniques, can provide a powerful and robust approach to link basic surface properties with electrocatalytic activity. ${ }^{20}$ We used Pt(111)-like thin films ${ }^{21}$ modified with monolayer amounts of $\mathrm{Cu}$ atoms ${ }^{22}$ as 
model objects. The position of the $\mathrm{Cu}$ atoms relative to the topmost Pt layer affects the surface properties ${ }^{23}$ and controls the electrocatalytic activity of the surface towards the oxygen reduction reaction (ORR). ${ }^{24-27}$ This combined SKP-SECM technique shows promise as a very sensitive tool to monitor changes in the surface status.

\section{Experimental}

\subsection{Preparation of model samples}

$\mathrm{Pt}(111)$-like thin films were prepared as reported elsewhere. ${ }^{\mathbf{2 1}}$ In brief, the preparation procedure involves physical vapour deposition of Pt thin films on $\mathrm{Si}(100)$ chips $(\mathrm{ca} .1 .5 \times 0.8 \mathrm{~cm})$ followed by flame annealing (10 periodic heating and cooling sequences, 10-15 s, with a butane/oxygen flame), electrochemical cleaning and a short heat treatment at $400{ }^{\circ} \mathrm{C}$ under controlled $\left(\mathrm{Ar} / \mathrm{H}_{2}\right.$ or $\left.\mathrm{Ar} / \mathrm{CO}\right)$ atmospheres. Before further modification the $\mathrm{Pt}(111)$-like thin films were voltammetrically cycled in 0.1 M Suprapur® $\mathrm{HClO}_{4}$ (Merck, Germany) aqueous solutions until stable cyclic voltammograms were obtained. The samples were then lifted so that approximately half of the surface was exposed to the electrolyte. The electrolyte-exposed surface was modified to produce either a $\mathrm{Cu}$ overlayer, $\mathrm{Cu} 3 \mathrm{D}$ clusters or $\mathrm{Cu}-$ $\mathrm{Pt}(111)$ near-surface alloys (Cu-Pt(111) NSAs as described in detail elsewhere ${ }^{22}$ ). To deposit pseudomorphic overlayers of $\mathrm{Cu}$, an underpotential $\mathrm{Cu}$ deposition was performed for $3 \mathrm{~min}$ at $0.33 \mathrm{~V}$ versus $\mathrm{RHE}$ from a $0.1 \mathrm{HClO}_{4}$ solution containing $2 \mathrm{mM}$ $\mathrm{Cu}^{2+}$. To produce $\mathrm{Cu}-\mathrm{Pt}(111)$ near-surface alloys samples with the deposited $\mathrm{Cu}$ overlayer were annealed at $400{ }^{\circ} \mathrm{C}$ in $\mathrm{Ar} / \mathrm{H}_{2}$ (6.0, $5 \% \mathrm{H}_{2}$, AirLiquide, Germany) atmosphere. Alternatively, clusters of $\mathrm{Cu}$ were deposited on top of the $\mathrm{Cu}$ overlayer $(0.2 \mathrm{~V}$ versus $\mathrm{RHE}$ ) to produce reference samples with a bulk $\mathrm{Cu}$ phase present at the surface. The amount of $\mathrm{Cu}$ on the surface was evaluated by calculating the charge passed through the system. It should be noted that electrochemical and thermal surface treatments were performed under conditions as similar as possible for all samples to minimize any effect caused by different surface pre-history.

\subsection{Electrochemical characterization of model samples}

Before each electrochemical experiment, all glassware was cleaned using "piranha" solution, a mixture of 96\% Suprapur® $\mathrm{H}_{2} \mathrm{SO}_{4}$ (Merck, Germany) and $30 \%$ TraceSelect ${ }^{\circledR}$ Ultra $\mathrm{H}_{2} \mathrm{O}_{2}$ (3: 1, Sigma-Aldrich, Germany) for 12 hours. To remove $\mathrm{SO}_{4}{ }^{2-}$ ions, multiple heating was combined with an ultrasonic treatment and rinsing with Siemens@ UltraClear water $(0.055 \mu \mathrm{S}$ $\mathrm{cm}^{-1}$, TOC content $<1 \mathrm{ppb}$ ). Solutions of $0.1 \mathrm{M} \mathrm{HClO}_{4}$ were prepared using Suprapur® $\mathrm{HClO}_{4}$ (Merck, Germany). Pt wire ( $D_{\text {out }}=1 \mathrm{~mm}$, Goodfellow, Germany) was used as counter electrode and a mercury-mercury sulphate (MMS, Schott, Germany) was used as reference electrode. During all electrochemical experiments the reference electrode was kept in a compartment separated from the working solution with a ceramic frit. For benchmark experiments, a commercial Pt(111) single crystal (diameter $0.5 \mathrm{~cm}$, oriented to $<0.1^{\circ}$, polished down to $30 \mathrm{~nm}$ roughness, obtained from Mateck, Germany) was used. Prior to each experiment it was pre-treated and modified as described in detail elsewhere ${ }^{29}$ using a specifically designed electrochemical cell for the preparation and characterisation of single crystal electrodes. ${ }^{27,30}$

\subsection{SKP measurements}

SKP-SECM tips were fabricated using glass-insulated Pt-disk microelectrodes made of borosilicate glass capillaries $\left(D_{\text {out }}=\right.$ $1.5 \mathrm{~mm}$, Hilgenberg, Germany) with a $25 \mu \mathrm{m}$ diameter Pt wire (Goodfellow, Germany) following a procedure described in detail elsewhere. ${ }^{\mathbf{1 0}}$ Before fabrication, glass capillaries were carefully sorted to ensure optimal fitting to the guide unit at the top of the SKP-SECM head. Prior to each experiment the SKPSECM tips were polished with different grades of alumina paste ( $3 \mu \mathrm{m}, 1 \mu \mathrm{m}, 0.3 \mu \mathrm{m}$ and $0.05 \mu \mathrm{m}$; LECO, USA) and subsequently ultrasonicated in acetone, 2-propanol and deionised water for $5 \mathrm{~min}$. After cleaning the electrodes were dried in an argon stream (6.0, AirLiquide, Germany).

Combined SKP-SECM experiments were performed in the recently described integrated SKP-SECM system. ${ }^{10}$ In the SKP mode of operation the SKP-SECM tip was oscillated with an oscillation amplitude of $9 \mu \mathrm{m}$ and an oscillation frequency of $1200 \mathrm{~Hz}$. Control of the tip-to-sample distance was performed using an additional alternating current (AC) signal $(6.73 \mathrm{~Hz}$, $0.25 \mathrm{~V}_{\mathrm{pp}}$ ). The corresponding AC current was demodulated by a lock-in amplifier and its value was kept constant by varying the position of the SKP tip above the sample surface. All SKP experiments were performed with a tip-to-sample distance of $5 \mu \mathrm{m}$. Model samples were fixed on the sample holder with a small drop of conductive silver filled lacquer (Electrodag ${ }^{\circledR}$ 1415M; Plano, Germany) to ensure stable mounting. An additional drop of the conductive lacquer was placed on the edge of model samples to establish direct electrical contact between the Pt metal film and the sample holder. During SKP measurements a piece of pure Ni (99.999\%; Goodfellow, Germany) was placed on the sample holder and was used as reference. All reported contact-potential differences (CPD) values are referred to the CPD value of pure $\mathrm{Ni}$.

\subsection{SECM measurements}

SECM experiments were carried out in a four-electrode cell configuration with the sample as working electrode one (WE 1), the SKP-SECM tip as working electrode two (WE 2), a Pt-wire as counter electrode and a $\mathrm{Ag} / \mathrm{AgCl} / 3 \mathrm{M} \mathrm{KCl}$ reference electrode. To minimize the presence of chloride ions in the electrochemical cell, a double junction reference electrode was used with the outer chamber filled with $0.1 \mathrm{M}$ Suprapur® $\mathrm{HClO}_{4}$ (Merck, Germany) solution. The electrochemical cell comprised of a glass tube ( $L=5 \mathrm{~mm}, D_{\text {in }}=6 \mathrm{~mm}$, Hilgenberg, Germany) placed on the sample surface and filled with $200 \mu \mathrm{L}$ of oxygen aerated 0.1 M Suprapur ${ }^{\circledR} \mathrm{HClO}_{4}$ solution. To establish a good contact between the glass tube and the sample surface, a thin layer of the hydrophobic polymer Sylgard 184 (Dow Corning, Germany) was deposited on the lower edge of the glass tube. The tip-tosample distance was adjusted to $2 \mu \mathrm{m}$ based on the negative feedback effect ${ }^{31}$ observed during approach to the sample 
surface $\left(E_{\mathrm{WE} 1}=\mathrm{OCP}, E_{\mathrm{WE} 2}=-0.6 \mathrm{~V}\right)$. Visualization of the catalytic ORR activity of the prepared sample was performed in the redox-competition mode of the SECM. ${ }^{32}$ A sequence of potential pulses was applied at the SKP-SECM tip $\left(P_{1}=0.65 \mathrm{~V}\right.$, $t_{1}=1 \mathrm{~s} ; P_{2}=1.5 \mathrm{~V}, t_{2}=0.2 \mathrm{~s} ; P_{3}=0.2 \mathrm{~V}, t_{3}=0.5 \mathrm{~s}$ ) while the sample was polarized at a constant potential of $0.175 \mathrm{~V}$ which was $25 \mathrm{mV}$ more cathodic than the OCP of the sample. To avoid any background current shift caused by an uncompensated tilt between the scanning plane of the SECM tip and the sample surface a software based tilt correction procedure was used. A PC in combination with a software module programmed in Microsoft Visual Basic 6.0 was used to control all system parameters, including data acquisition. To eliminate electrical noise all measurements were carried out in a grounded Faraday cage.

\section{Results and discussion}

Fig. 1 schematically represents the modification procedures for the fabrication of a $\mathrm{Pt}(111)$-like thin film and the $\mathrm{Cu}-\mathrm{Pt}(111)$ near-surface alloy (NSA). A Cu-Pt(111) NSA comprises approximately $2 / 3$ monolayer (ML) of Cu located predominantly in the second Pt layer. ${ }^{24,28}$ This system is known to be significantly more active towards the oxygen reduction reaction than pure $\mathrm{Pt}(111),{ }^{22,24}$ making it a good model catalyst for the envisaged study.

To evaluate the electrochemical and electrocatalytic behaviour of $\mathrm{Cu}-\mathrm{Pt}(111)$ modified thin films, voltammetric experiments were performed and the results were compared with those obtained using commercial benchmark Pt(111) single crystal electrodes. Fig. 2A compares cyclic voltammograms obtained in Ar-saturated $0.1 \mathrm{M} \mathrm{HClO}_{4}$ for the $\mathrm{Pt}(111)$ single crystal and the corresponding $\mathrm{Cu}-\mathrm{Pt}(111)$ NSA prepared using the same single crystal electrode. The voltammograms are very close to those characteristic of the well-ordered Pt(111) surfaces and $\mathrm{Cu}-\mathrm{Pt}(111)$ NSAs reported earlier. The oxygen reduction activity corresponds to that of bulk $\mathrm{Pt}(111)^{33}$ and $\mathrm{Cu}-\mathrm{Pt}(111)$ NSAs reported previously (Fig. 2B). ${ }^{24}$ Fig. $2 \mathrm{C}$ and D also show the

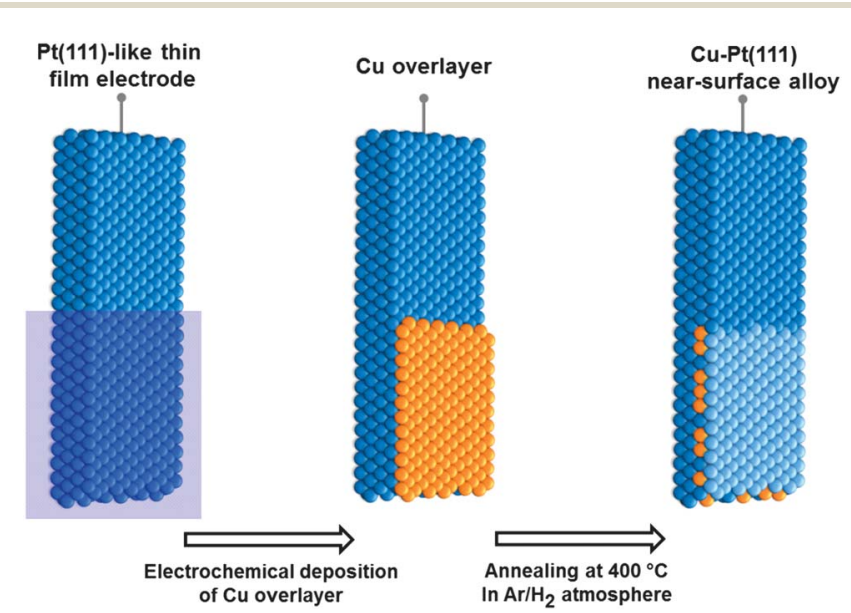

Fig. 1 Schematics illustrating the preparation of the model $\mathrm{Cu}-\mathrm{Pt}(111)$ samples for SKP-SECM measurements.
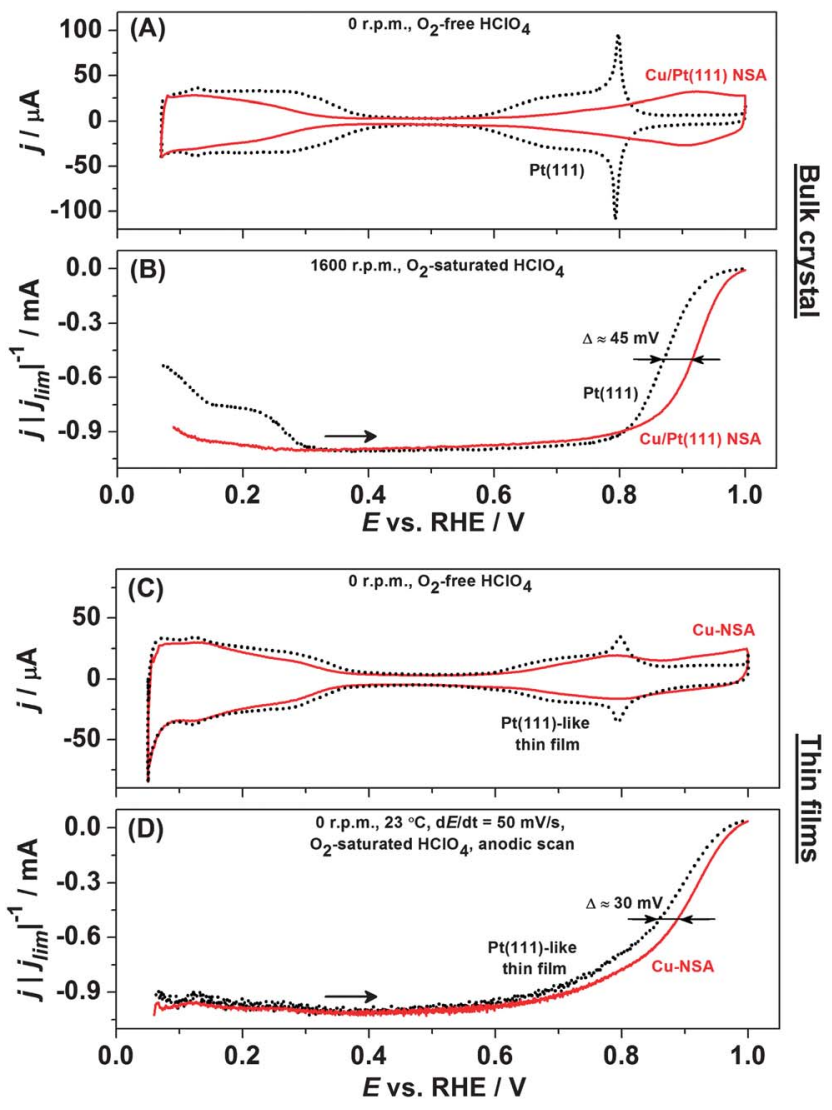

Fig. 2 Voltammetric characterisation of the Pt(111) single crystal and $\mathrm{Cu}-\mathrm{Pt}(111)$ NSA prepared using a Pt(111) single crystal surface in Arsaturated $(A)$ and $\mathrm{O}_{2}$-saturated $(B) 0.1 \mathrm{M} \mathrm{HClO}_{4}$. The results of similar experiments for the $\mathrm{Pt}(111)$-like thin film and $\mathrm{Cu}-\mathrm{Pt}(111) \mathrm{NSA}$ thin film (denoted as Cu-NSA) samples are shown in (C) and (D), respectively.

corresponding data for the model Pt(111)-like thin film and $\mathrm{Cu}-$ Pt(111) NSA thin film samples.

Due to the fact that the surface of the thin film samples are $a$ priori more defective than the surface of the bulk single crystals, the characteristic features in the corresponding voltammograms (e.g. the peaks at $\sim 0.8 \mathrm{~V} \mathrm{RHE)} \mathrm{are} \mathrm{less} \mathrm{pronounced} \mathrm{and}$ the activity towards ORR is slightly lower for $\mathrm{Cu}-\mathrm{Pt}(111)$ thin films. ${ }^{22}$ Nevertheless, the electrochemical and, particularly, electrocatalytic properties of both thin film samples are fairly close to those obtained for the benchmark single crystal surfaces making them good model objects for the evaluation of the SKP-SECM approach for detecting slight changes in surface compositions.

Fig. 3 shows the result of the SKP measurement for $\mathrm{Cu}$ modified Pt(111)-like thin film model samples. The typical CPD values as measured by SKP above the unmodified Pt(111)-like thin film and modified $\mathrm{Cu}-\mathrm{Pt}(111)$ NSA regions of the model sample (Fig. 3A) differ by approximately $150 \mathrm{mV}$. The modified NSA-region systematically reveals higher CPD values as compared to the unmodified surface. In contrast, the $\mathrm{Cu}$ overlayer (where $\mathrm{Cu}$ atoms constitute the topmost surface layer) showed the opposite trend: the CPD values are systematically lower by approximately $100 \mathrm{mV}$ in the $\mathrm{Cu}$ region compared to 

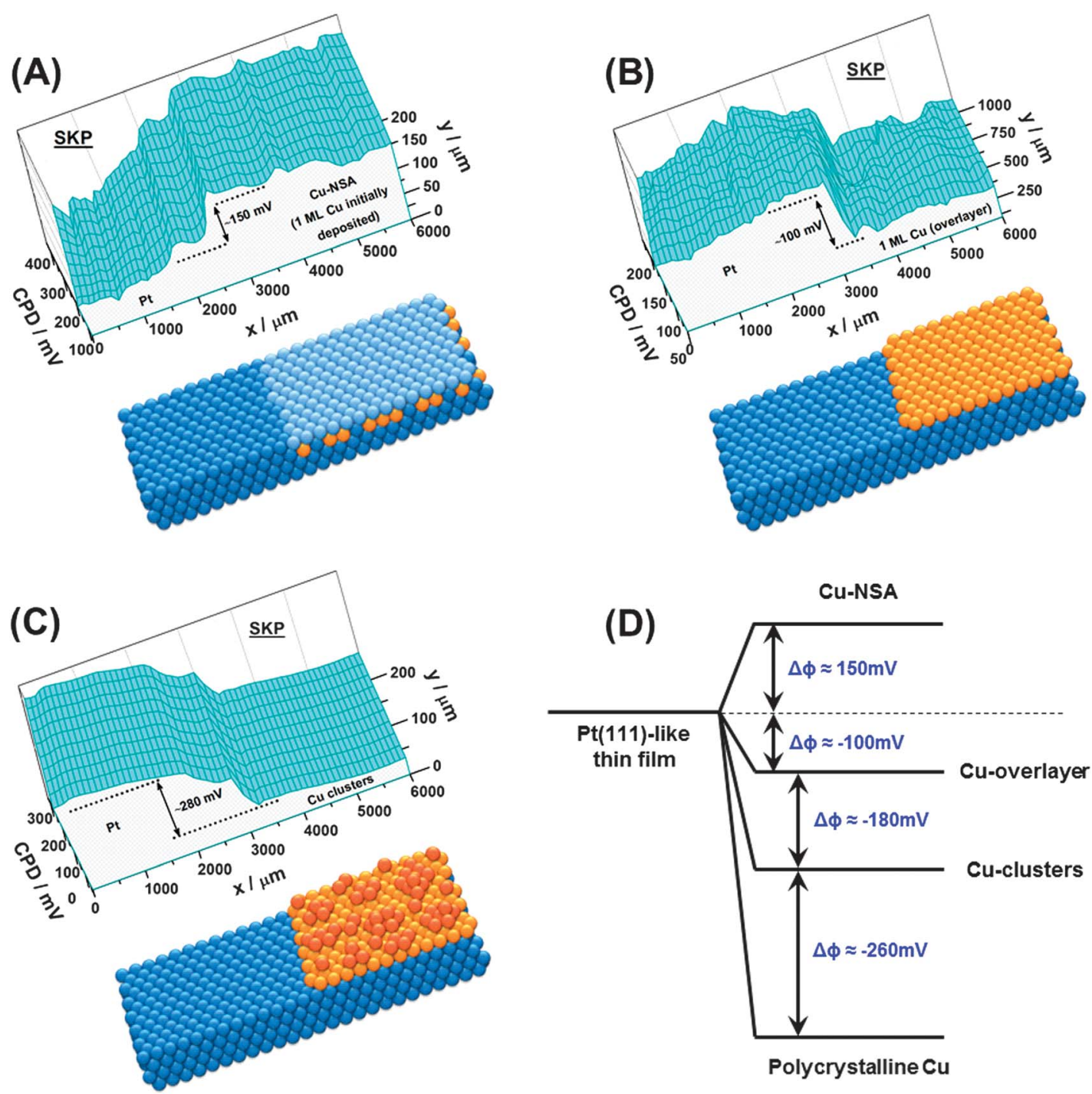

Fig. 3 SKP characterisation of a (A) Pt(111)-like thin film samples partially modified with Cu-Pt(111) NSA, (B) Pt(111)-like thin film sample partially modified with $1 \mathrm{ML}$ of $\mathrm{Cu}$ and (C) Pt(111)-like thin film sample partially modified with 3D Cu clusters. Differences in the corresponding CPD values are shown as a function of the spatial coordinates. Fig. 3D represents a schematic diagram showing the relative differences in the measured CPD values between the unmodified and modified surfaces.

the unmodified Pt(111)-like thin film surface (Fig. 3B). Additionally, electrochemically deposited $\mathrm{Cu}$ clusters further decrease the CPD values by approximately $180 \mathrm{mV}$ compared to the overlayer (Fig. 3C and D). Finally, in a control experiment using a bulk polycrystalline $\mathrm{Cu}$ sample the measured CPD values were approximately $260 \mathrm{mV}$ lower than that of the $3 \mathrm{D} \mathrm{Cu}-$ cluster surface (Fig. 3D).

Comparing the data for the different modified surfaces in Fig. 3, one can conclude that the CPD values measured using SKP are very sensitive to the position and status of the $\mathrm{Cu}$ atoms at the surface, even though the measurements are performed under almost ambient conditions. It should be noted that the measured SKP response likely has contributions that originate from various surface structures, adsorbed water layers, the composition of the gas atmosphere etc., however, when the CPD values are compared to the unmodified Pt(111)-like thin film reference surface under the same conditions, one can reasonably assume that this relative change is closely related to the variation of the work function across the surface. In principle, the measured CPD values can be used as an analytical signal by itself to provide basic information about the surface status. If necessary, quantum chemical approaches such as particularly DFT calculations may provide an independent benchmark value for a wide range of virtually any structure and composition formed at the surface as shown previously. ${ }^{34}$

The SKP data presented in Fig. 3 also reveal a non-trivial trend in the change of the CPD values as a function of the composition and relative position of $\mathrm{Cu}$ ad-atoms. This nontrivial, but in most cases theoretically predictable behaviour of the CPD values and its correlation with the calculated work function changes make SKP measurements a good probe to explore surface properties on a microscopic level. 
While theory assisted SKP measurements can provide key information about surface properties, e.g. revealing microscopic domains with different surface status, complementary SKPSECM measurements can help to link them with the electrocatalytic activity measured using the SECM technique.

Fig. 4A schematically represents the SECM experiment performed in the redox-competition mode on the $\mathrm{Pt}(111)$-like thin film sample partially (right side in Fig. 4A) modified with a $\mathrm{Cu}-$ Pt(111) NSA. Fig. 4B shows the results of combined SKP-SECM investigation of the model $\mathrm{Pt}(111)$-like thin film sample partially modified with $\mathrm{Cu}-\mathrm{Pt}(111) \mathrm{NSA}$ ( $\sim 1 / 2$ of the sample), comparing the modified and unmodified parts of the surface.

To avoid complete depletion of oxygen in the gap between the SKP-SECM tip and the sample, a potential pulse profile consisting of 3 pulses was applied at the tip while the sample was polarized at a constant potential of $0.175 \mathrm{~V}$ which was $25 \mathrm{mV}$ more cathodic than the OCP of the sample. The first potential pulse $\left(P_{1}=0.65 \mathrm{~V}, t_{1}=1 \mathrm{~s}\right)$ is a conditioning potential applied to restore diffusional equilibrium after movement of the SECM tip during scanning. The second potential pulse $\left(P_{2}=\right.$ $1.5 \mathrm{~V}, t_{2}=0.2 \mathrm{~s}$ ) is the injection pulse which leads to oxidation

(A)

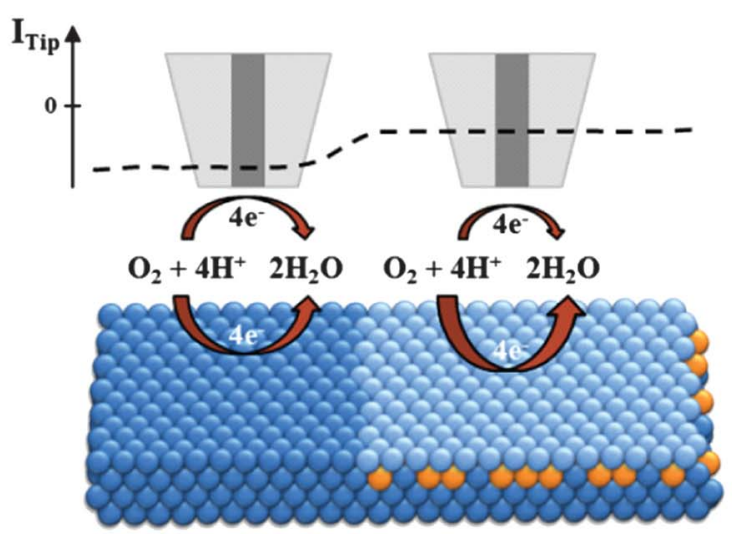

(B)

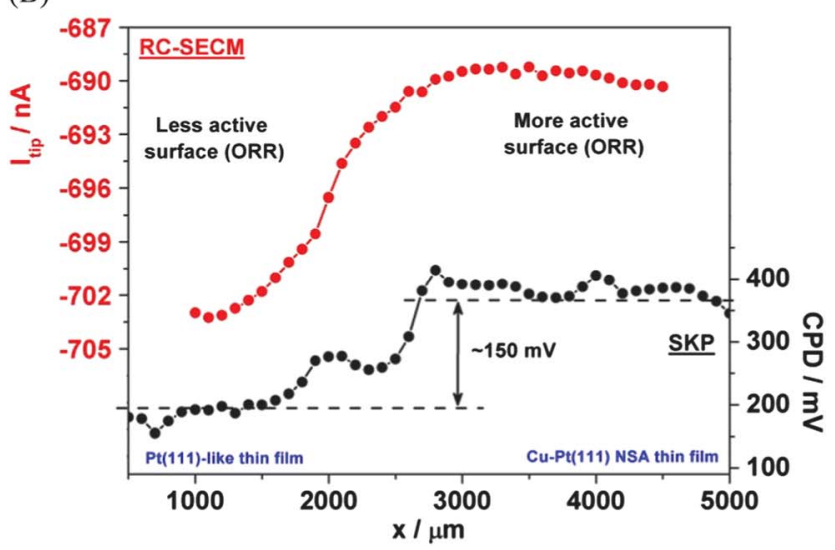

Fig. 4 Schematic representation of the SECM experiment performed in the redox-competition mode of the SECM on the Pt(111)-like thin film sample partially $(1 / 2$, right side) modified with a $\mathrm{Cu}-\mathrm{Pt}(111) \mathrm{NSA}(\mathrm{A})$. (B) represents a typical SKP and SECM line profiles sequentially recorded over exactly the same sample surface. of water and injection of oxygen into the gap between tip and sample. The third potential pulse $\left(P_{3}=0.2 \mathrm{~V}, t_{3}=0.5 \mathrm{~s}\right)$ is the actual measurement pulse which is sufficiently cathodic to invoke oxygen reduction at the tip. As the sample was continuously polarized at a potential sufficiently cathodic for oxygen reduction, application of the measurement pulse $P_{3}$ leads to oxygen reduction at the tip and hence to a competition between tip and sample for dissolved $\mathrm{O}_{2}$. With increased local ORR activity of the sample, the remaining amount of $\mathrm{O}_{2}$ which is accessible for the tip decreases leading to smaller cathodic tip currents over areas with higher ORR activity. $\mathrm{Cu}$ atoms located in the second layer of the Cu-Pt(111) NSA thin film sample obviously modify the electronic structure of the topmost Pt layer. The concomitant change in the binding energies for reaction intermediates leads to an increasing electrocatalytic activity. Particularly, as shown in Fig. 2, Cu-Pt(111) NSA thin film exhibit significantly higher catalytic activity towards ORR as compared to the unmodified Pt(111)-like thin film surface. Higher cathodic currents observed over the Pt(111)-like thin film (Fig. 4B) therefore clearly indicate the higher catalytic ORR activity of the $\mathrm{Cu}-\mathrm{Pt}(111)$ NSA thin film surface as compared to that of the unmodified $\mathrm{Pt}(111)$-like thin film surface.

\section{Conclusions}

Combined sequential SKP and SECM measurements above exactly the same surface area appeared to be a very sensitive tool for the detection of relatively small changes of the basic surface properties and correlation of these with electrocatalytic activity. The model experiments demonstrated that the relative CPD between the unmodified and Cu-modified $\mathrm{Pt}(111)$ thin film surfaces is very sensitive to the exact position and status of $\mathrm{Cu}$ atoms. As SKP-responses can be accurately assessed using quantum chemical approaches, the combined SKP-SECM system offers a valuable insight into the origin of the observed electrocatalytic activity and an affordable tool to carefully evaluate novel functional materials.

\section{Acknowledgements}

Financial support by the EU and the state NRW in the framework of the HighTech. NRW program is gratefully acknowledged. A.S.B. and W.S. additionally acknowledge financial support in the framework of Helmholtz-Energie-Allianz "Stationäre elektrochemische Speicher und Wandler" (HA-E0002) and the Cluster of Excellence RESOLV (EXC 1069) funded by the DFG (Deutsche Forschungsgemeinschaft).

\section{References}

1 J. K. Nørskov, T. Bligaard, J. Rossmeisl and C. H. Christensen, Nat. Chem., 2009, 1, 37.

2 F. Calle-Vallejo and M. T. M. Koper, Electrochim. Acta, 2012, 84, 3 .

3 M. T. M. Koper, Nanoscale, 2011, 3, 2054.

4 F. Zaera, Chem. Rev., 2012, 112, 2920. 
5 C. Batchelor-McAuley, E. J. F. Dickinson, N. V. Rees, K. E. Toghill and R. G. Compton, Anal. Chem., 2012, 84, 669.

6 A. S. Bandarenka and M. T. M. Koper, J. Catal., 2013, DOI: 10.1016/j.jcat.2013.05.006).

7 K. Eckhard and W. Schuhmann, Analyst, 2008, 133, 1486.

8 K. Eckhard, T. Erichsen, M. Stratmann and W. Schuhmann, Chem.-Eur. J., 2008, 14, 3968.

9 A. S. Bandarenka, K. Eckhard, A. Maljusch and W. Schuhmann, Anal. Chem., 2013, 85, 2443.

10 A. Maljusch, B. Schönberger, A. Lindner, M. Stratmann, M. Rohwerder and W. Schuhmann, Anal. Chem., 2011, 83, 6114.

11 K. Eckhard and W. Schuhmann, Electrochim. Acta, 2007, 53, 1164.

12 C. Senöz, A. Maljusch, M. Rohwerder and W. Schuhmann, Electroanalysis, 2012, 24, 239.

13 A. Maljusch, C. Senöz, M. Rohwerder and W. Schuhmann, Electrochim. Acta, 2012, 82, 339.

14 K. Eckhard, C. Kranz, H. Shin, B. Mozaikoff and W. Schuhmann, Anal. Chem., 2007, 79, 5435.

15 K. J. P. Schouten, Y. Kwon, C. J. M. van der Ham, Z. Qin and M. T. M. Koper, Chem. Sci., 2011, 2, 1902.

16 M. Rohwerder and F. Turcu, Electrochim. Acta, 2007, 53, 290. 17 M. Stratmann and H. Streckel, Corros. Sci., 1990, 30, 697.

18 C. J. Fall, N. Binggeli and A. Baldereschi, J. Phys.: Condens. Matter, 1999, 11, 2689.

19 L. Kornblum, P. Shekhter, Y. Slovatizky, Y. Amouyal and M. Eizenberg, Phys. Rev. B: Condens. Matter Mater. Phys, 2012, 86, 125305.

20 S. Amemiya, A. J. Bard, F.-R. F. Fan, M. V. Mirkin and P. R. Unwin, Annu. Rev. Anal. Chem., 2008, 1, 95.

21 A. Maljusch, J. Henry, W. Schuhmann and A. S. Bondarenko, Electrochem. Commun., 2012, 16, 88.
22 J. B. Henry, A. Maljusch, M. Huang, W. Schuhmann and A. S. Bondarenko, ACS Catal., 2012, 2, 1457.

23 A. S. Bandarenka, A. S. Varela, M. Karamad, F. Calle-Vallejo, L. Bech, F. J. Perez-Alonso, J. Rossmeisl, I. E. L. Stephens and I. Chorkendorff, Angew. Chem., Int. Ed., 2012, 51, 11845.

24 I. E. L. Stephens, A. S. Bondarenko, F. J. Perez-Alonso, F. Calle-Vallejo, L. Bech, T. P. Johansson, A. K. Jepsen, R. Frydendal, B. P. Knudsen, J. Rossmeisl and I. Chorkendorff, J. Am. Chem. Soc., 2011, 133, 5485.

25 A. S. Bondarenko, I. E. L. Stephens, L. Bech and I. Chorkendorff, Electrochim. Acta, 2012, 82, 517.

26 J. B. Henry, A. Maljusch, J. Tymoczko, W. Schuhmann and A. S. Bandarenka, Electrochim. Acta, 2013, DOI: 10.1016/ j.electacta.2012.11.139).

27 J. Tymoczko, W. Schuhmann and A. S. Bandarenka, Phys. Chem. Chem. Phys., 2013, 15, 12998-13004.

28 K. J. Andersson, F. Calle-Vallejo, J. Rossmeisl and I. J. Chorkendorff, J. Am. Chem. Soc., 2009, 131, 2404.

29 A. S. Bondarenko, I. E. L. Stephens, H. A. Hansen, F. J. Perez-Alonso, V. Tripkovic, T. P. Johansson, J. Rossmeisl, J. K. Nørskov and I. Chorkendorff, Langmuir, 2011, 27, 2058.

30 A. S. Bondarenko, I. E. L. Stephens and I. Chorkendorff, Electrochem. Commun., 2012, 23, 33.

31 J. Kwak and A. J. Bard, Anal. Chem., 1989, 61, 1221.

32 K. Eckhard, X. Chen, F. Turcu and W. Schuhmann, Phys. Chem. Chem. Phys., 2006, 8, 5359.

33 A. Berna, V. Clement and J. M. Feliu, Electrochem. Commun., 2007, 9, 2789.

34 M. Huang, A. Maljusch, F. Calle-Valejo, J. B. Henry, M. T. M. Koper, W. Schuhmann and A. S. Bandarenka, $R S C$ $A d v .$, 2013, 3, 21648. 